\section{Combinatorial chemistry}

\section{Scavenger resins}

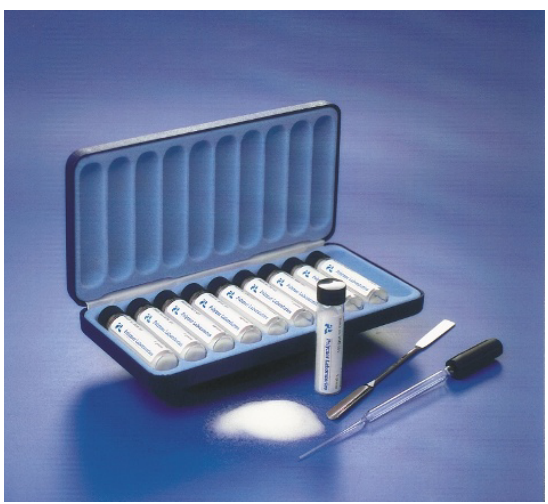

Polymer Laboratories' (Church Stretton, UK) StratoSpheres range of particles for combinatorial chemistry and peptide synthesis now includes high-load scavenger resins and solid-supported reagents, available in a kit comprising $5 \mathrm{~g}$ each of the following 10 resin types: benzaldehyde, diethylenetriamine, ethylenediamine, hydroxymethylstyrene, morpholine, isocyanate, piperidine, piperazine, acrylate, and sulfonic acid. Benefits include higher chemical purity and reproducible functionality, tightly controlled particle size distributions to improve filtration rates, and higher resin loadings for increased yield and higher sensitivity with techniques such as IR and NMR.

http://www.polymerlabs.com

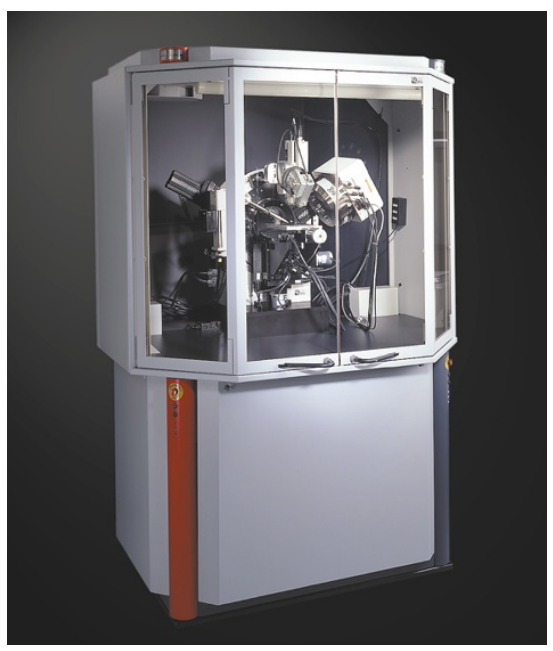

\section{X-ray diffraction}

The D8 Discover with GADDS, from Bruker AXS (Madison, WI), provides a nondestructive solution for rapid screening of materials libraries. It incorporates a unique twodimensional detector and XYZ stage for precision mapping, and a laser/video system for easy sample alignment and positioning. GADDS software makes this system ideal for combinatorial analysis.

http://www.bruker-axs.com

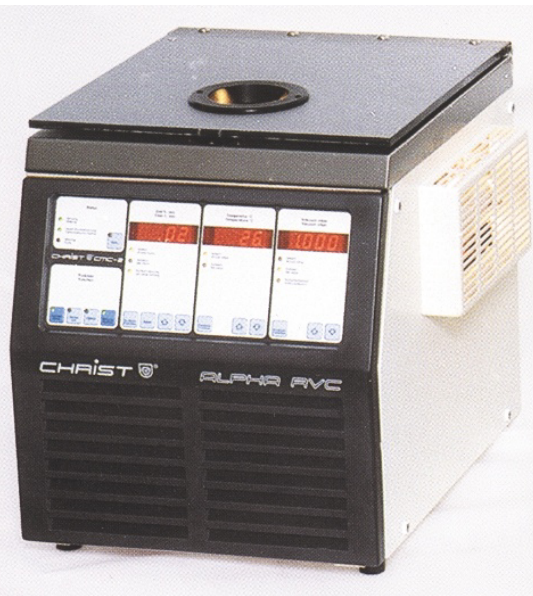

\section{Vacuum concentrator}

The Alpha-RVC "IR" is a benchtop rotational vacuum concentrator for high-speed evaporation, ideal for use in combinatorial chemistry and high-throughput screening. A vacuum measurement sensor in combination with a chemical-resistant diaphragm pump allows accurate regulation of the vacuum, essential for the rapid extraction of highboiling-point solvents such as dimethyl sulfoxide, trifluoroacetic acid, and dimethyl formamide. The Alpha-RVC "IR" is available from BioPro (Farmingdale, NY) and Martin Christ (Osterode, Germany).

http://www.biopro.com

http://www.martinchrist.de

\section{Enzymes \& reagents}

\section{PNA probes}

Peptide nucleic acid (PNA) probes are DNA mimics with higher specificity and more rapid hybridization kinetics. PNA Micro Dx probes and reagent kits are available from Boston Probes (Bedford, MA), including species-specific probes targeting ribosomal RNA of Salmonella enterica, Staphylococcus aureus, and Escherichia coli, as well as universal PNA probes for eubacteria and eukaryotes. Optimized reagent kits provide an attractive assay format for fluorescent in situ hybridization or dotblot analysis.

$h t t p: / / w w w . b o s t o n p r o b e s . c o m$

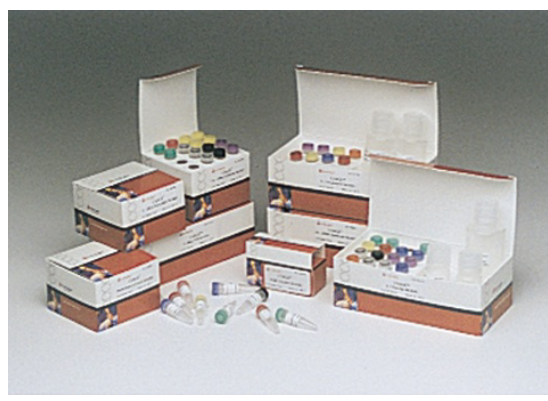

\section{Gene discovery kit}

Invitrogen's (Carlsbad, CA) SAGE (serial analysis of gene expression) technology allows for comprehensive genome-wide expression analysis, and the I-SAGE kit is designed to make constructing SAGE libraries convenient. The kit includes reagents organized into nine modules for each step of the SAGE process, including two complementary DNA synthesis modules, a cleavage module, a ditag formation module, a ditag amplification module, a concatemer module, an Nla III module, a performance check module, the Zero Background Cloning Kit, and One Shot TOP10 Electrocomp E. coli. All reagents are guaranteed stable for six months when properly stored.

http://www.invitrogen.com

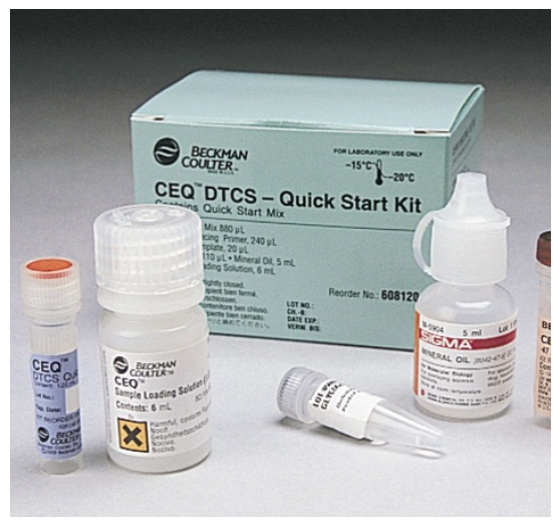

\section{Sequencing reaction setup}

Made for use with the CEQ 2000XL DNA sequencing system, Beckman Coulter (Fullerton, CA) introduces the DTCS Quick Start kit for simplified dye terminator cycle sequencing reaction setup. The kit includes thermostable polymerase and pyrophosphatase, reaction buffer, control primer and template, ddNTPs with WellRED dyes, dNTP, and other reagents that reduce the number of pipetting steps from 10 to 4 , and uses larger transfer volumes to reduce pipetting error.

http://www.beckmancoulter.com 